\title{
Prognostic value of 5-microRNA based signature in T2-T3N0 colon cancer
}

\author{
Maciej Bobowicz ${ }^{1} \cdot$ Marcin Skrzypski $^{2}$ (1) Piotr Czapiewski ${ }^{3} \cdot$ Michał Marczyk $^{4} \cdot$ \\ Agnieszka Maciejewska ${ }^{5}$ - Michał Jankowski ${ }^{6}$ - Anna Szulgo-Paczkowska ${ }^{7}$. \\ Wojciech Zegarski ${ }^{6} \cdot$ Ryszard Pawłowski $^{5} \cdot$ Joanna Polańska $^{4} \cdot$ Wojciech Biernat $^{3}$. \\ Janusz Jaśkiewicz $^{1} \cdot$ Jacek Jassem $^{2}$
}

Received: 28 January 2016/ Accepted: 6 July 2016/Published online: 2 August 2016

(c) The Author(s) 2016. This article is published with open access at Springerlink.com

\begin{abstract}
The role of adjuvant chemotherapy in stage T2T3NO colon cancer (CC) is controversial and there are currently no reliable factors allowing for individual selection of patients with high risk of relapse for such therapy. We searched for microRNA-based signature with prognostic significance in this group. We assessed by qRT-PCR expression of 754 microRNAs (miRNAs) in tumour samples from 85 stage pT2-3N0 CC patients treated with surgery alone. MiRNA expression was compared between two groups of patients: 40 and 45 patients who did and did not develop distant metastases after resection, respectively.
\end{abstract}

Maciej Bobowicz and Marcin Skrzypski equally contributed to the presented study.

Electronic supplementary material The online version of this article (doi:10.1007/s10585-016-9810-1) contains supplementary material, which is available to authorized users.

Marcin Skrzypski

mskrzypski@gumed.edu.pl

1 Department of Surgical Oncology, Medical University of Gdansk, Gdansk, Poland

2 Department of Oncology and Radiotherapy, Medical University of Gdansk, 7 Dębinki St., 80-211 Gdańsk, Poland

3 Department of Pathomorfology, Medical University of Gdansk, Gdańsk, Poland

4 Institute of Automatic Control, Data Mining Group, Silesian University of Technology, Gliwice, Poland

5 Institute of Forensic Medicine, Medical University of Gdansk, Gdańsk, Poland

6 Department and Clinic of Oncologic Surgery, Collegium Medicum, Nicolaus Copernicus University, Bydgoszcz, Poland

7 Department of Clinical Oncology, Centre of Oncology, Bydgoszcz, Poland
Additionally, miRNA expression was compared between $\mathrm{CC}$ and normal colon mucosa samples and between the mismatch repair (MMR) competent and deficient tumours. Low expression of miR-1300 and miR-939 was significantly correlated with shorter distant metastasis-free survival (DMFS) in Cox univariate analysis (p.adjusted $=0.049$ ). The expression signature of five miRNAs (miR-1296, miR-135b, miR-539, miR-572 and miR-185) was found to be prognostic [ $p=1.28 \mathrm{E}-07$, HR 8.4 (95 \% CI: 3.81-18.52)] for DMFS and cross-validated in a leave-one-out analysis, with the sensitivity and specificity of 74 and $78 \%$, respectively. The expression of miR-592 was significantly associated with the MMR status (p.adjusted $<0.01$ ). The expression of several novel miRNAs were found to be tumour specific, e.g. miR-888, miR523, miR-18b, miR-302a, miR-423-5p, miR-582-3p $(\mathrm{p}<0.05)$. We developed a miRNA expression signature that may be predictive for the risk of distant relapse in early stage CC and confirmed previously reported association between miR-592 expression and MMR status.

Keywords microRNA expression · colon cancer . metastasis $\cdot$ prognostic marker $\cdot \operatorname{miR}-1300 \cdot \operatorname{miR}-939$

\section{Background}

Colon cancer (CC) is the fourth greatest cause of cancerrelated deaths worldwide [1]. Around 10-20\% of stage II patients (pT3/T4N0) will develop distant metastases after curative resection [2]. Currently, adjuvant chemotherapy is recommended in T4N0 patients with additional clinicopathological adverse features [2]. The use of adjuvant chemotherapy in stage II CC patients results in the improvement of the 5-year survival by a mere $2-4 \%[3,4]$. 
pT2N0 and pT3N0 patients are usually not prescribed adjuvant chemotherapy, even though a proportion of them will develop distant metastases. Hence, the identification of patients with high risk of dissemination in this subset may optimise the use of adjuvant therapies.

Molecular traits have been explored as a potential prognostication tool for CC. Mutations in oncogenes (e.g. TP53, B-raf or $K$-ras) have been shown to carry prognostic information, however they are insufficient for individual patient selection to adjuvant chemotherapy [5-9]. Microsatellite instability (MSI-H) has been repeatedly shown to correlate with CC prognosis $[10,11]$. However MMR deficiency (dMMR) occurs in only around $15 \%$ of sporadic CCs [12], and therefore remains irrelevant to the majority of CC cases.

Recently, prognostic gene-expression signatures have been developed in CC, among which Colon OncotypeDx [13] and the six-cluster gene expression ColoPrint [14] have been extensively validated in retrospective series. However, stage II-III CC patients identified by these classifiers as carrying 'high risk' have an approximately $60 \%$ chance of 5-year survival with surgery alone. Therefore, a search for more specific prognostic classifiers is warranted.

MicroRNAs (miRNAs) are short, non-coding RNAs that play key roles in cancer cell [15]. These molecules regulate gene expression by binding to the target sequences of mRNA, which results in hindered translation [16]. It is estimated that miRNAs tune the expression of more than $30 \%$ of human genes [17]. Notably, miRNA is stable in formalin-fixed paraffin-embedded (FFPE) samples, and therefore their analysis is not significantly affected by the storing time of tissue samples [18].

Recently, Zhang et al. [19] demonstrated the prognostic value of 5-miRNA-expression signature in stage II CC, and validated it in sizeable validation cohorts. A populationbased translational study by Slattery et al. [20] confirmed the prognostic value of several other miRNAs. In another study, miR-362-3p was positively validated as prognostic in CC [21]. However, next generation sequencing-based study using fresh frozen material did not detect any prognostic miRNAs [22].

In the current study, we explored prognostic value of miRNA expression in stage T2-T3N0 CC, with the aim of developing a multi-miRNA prognostic expression signature. To this end, we assessed the expression of 754 miRNAs with qRT-PCR in FFPE samples from patients with or without distant relapse. Additionally, we investigated miRNA expression associations with microsatellite instability [23] and compared expression of miRNA between $\mathrm{CC}$ and normal colon mucosa (NCM) samples.

\section{Methods}

\section{Patients}

This study was approved by the Ethics Committee of the Medical University of Gdańsk. The study subjects were pT2-3N0 CC patients who underwent curative resection between 2001 and 2011, and either did or did not develop distant metastases. No-relapse group comprised patients with relapse-free survival of at least 4 years. Both groups were matched by major clinico-pathological features. Patients who developed isolated local or nodal recurrence were excluded. All patients underwent pathologically confirmed complete hemicolectomy or sigmoidectomy. To avoid confounding effect of occult nodal disease (active lymphatic spread), all cases were required to have had at least 12 lymph nodes excised. None of the patients received preoperative or postoperative chemotherapy.

\section{Pathological and molecular analyses}

The study material was archival FFPE blocks containing primary tumour samples obtained at resection. All cases were reviewed using hematoxylin-eosin (HE) staining independently by two pathologists (PC and WB) to confirm $\mathrm{CC}$ diagnosis, in accordance with the WHO criteria. One slice from each block was HE stained and reviewed, and the block with the highest percentage of cancer tissue was chosen for molecular analysis. To further decrease the content of non-carcinomatous tissue, surrounding nonneoplastic component (normal mucosa, muscular layer, pericolic fat and necrotic tissue) was removed. The macrodissected blocks were required to obtain at least $80 \%$ of viable tumour tissue. To avoid cross-contamination, the blades used for macrodissection and for slice cutting were changed after each case.

Four slices of $20 \mu \mathrm{m}$ each were cut for total RNA isolation with RecoverAll Kit (Ambion). The concentration of RNA was assessed in NanoDrop ${ }^{\circledR}$. Reverse transcription was carried out with $750 \mathrm{ng}$ of RNA with TaqMan MiRNA RT kit (Applied Biosystems) and pools A and B of stem-loop primers (Megaplex ${ }^{\mathrm{TM}}$ Primer Pools, Human Pools Set v3.0, Applied Biosystems), in accordance with the manufacturer's instructions. These pools contain primers specific for 377 different miRNAs, therefore to obtain cDNA for $754 \mathrm{miR}$ NAs, in each sample reverse transcription was carried out twice-with pool A and pool B primers. cDNA was quantified by qRT-PCR, with the use of miRNAs specific primer pairs, fluorescent TaqMan probes and polymerase with $5^{\prime}$ nuclease activity, in microfluidic cards (TaqMan ${ }^{\circledR}$ Array Microfluidic Cards, Applied Biosystems) in HT 7900 cycler (Applied Biosystems), with reaction conditions in 
accordance with manufacturer's instructions (Applied Biosystems). Raw expression results (Ct values) were obtained through SDS.2.1 (Applied Biosystems) software.

All tumour slides were also assessed for expression of MMR proteins by immunohistochemistry (IHC) in tissue microarrays (TMAs) that included two cores of $1.5 \mathrm{~mm}$ diameter. After cutting $4 \mu \mathrm{m}$ slides, the TMAs were stained in Dako autostainer for MLH1 (clone ES05, Dako, ready to use), MSH2 (G219-1129, Cell Marque, 1:200) and MSH6 (clone EP49, Dako, ready to use). The complete lack of IHC reaction for MLH1, MSH2 or MSH6 in cancer tissue, with retained expression in the surrounding stroma, was considered an indicator of microsatellite instability (MSI).

The NCM samples were obtained from the free surgical margins of the pathological specimen from which the CC sample was acquired.

\section{Statistical analysis}

The primary clinical endpoint was distant metastasis-free survival (DMFS). The number of miRNAs that were expressed in at least $25,50,75$ and $100 \%$ of the samples in both analysed groups ('relapsed' or 'non-relapsed') were calculated. MiRNAs with no amplification signal $(\mathrm{Ct} \geq 40)$ in less than $5 \%$ of samples were included in the prognostic analyses. The rationale for this threshold for expression positivity is provided in the Supplementary material. Undetermined values of expressions $(\mathrm{Ct} \geq 40)$ for this set of miRNAs were imputed by EM-based model of the missing data mechanism [24]. The individual $\mathrm{Ct}$ values for target miRNAs were normalised against the geometrical mean of the Ct values of U6 RNA, RNU44 and RNU48, and nine most stably expressed miRNAs that were determined with use of the NormFinder application (Appendix A, Table 1 in Supplementary material) [25]. Expression of miRNAs obtained with the $2^{-(\Delta \mathrm{Ct})}$ method $[26,27]$ was used to calculate fold change and its $95 \%$ confidence interval. Expression of miRNAs obtained with the $\Delta \mathrm{Ct}$ method was used in subsequent analysis. Lilliefors test was used for the verification of the hypothesis on normality of the analysed signal. Univariate analyses were performed to examine the normalised miRNA expression changes between both patient groups with the use of non-parametric Mann-Whitney test and univariate Cox regression, with DMFS information included. P values were adjusted for multiple hypotheses testing using Benjamini-Hochberg algorithm. All statistical inferences were performed at significance level equal to 0.05 . The DMFS curves were generated using Kaplan-Meier method. The clinical relevance of individual miRNAs was further tested in Cox multivariate models that included miRNA expression (as $-\Delta \mathrm{Ct}$ ), the $\mathrm{T}$ stage and the histological grade. The negative $\Delta \mathrm{Ct}$ allowed for the intuitive interpretation of resulting HR values, e.g. for the cases where the high miRNA expression was associated with the high risk of relapse, the obtained HR value was $>1$. The $\mathrm{T}$ feature was projected to a binary status (pT2 vs. pT3) and the grade was included with the values of 1,2 or 3 , respectively.

Multivariable logistic regression model was constructed to find an expression signature that describes the dependence between miRNA expression and recurrence status. For each patient recurrence score (RS) was estimated as prediction of logistic regression model. Given the large number of potential explanatory variables, regression model was limited to variables that were associated with $p$ values $\leq 0.2$ in the univariate analyses [28]. The number of model predictors was found using forward selection scheme, with manual tuning based on Bayesian information criterion, $\mathrm{R}^{2}$ and a $\mathrm{p}$ value of likelihood ratio test indicating difference from a smaller model. Contribution of an individual predictor was measured using the Wald test. Optimal threshold for RS was found by maximizing positive predictive value (PPV) with the constraint on negative predictive value (NPV) higher than 0.8. Additionally, regularised parameters of estimated model were obtained by using LASSO regression [29]. Prognostic capabilities of the obtained model were checked by internal leave-one-out cross-validation. The clinical relevance of the signature was further tested in Cox multivariate models that included $\mathrm{T}$ stage and histological grade. The study including 85 patients, comprising 40 patients with cancer recurrence'events', and 45 without disease recurrence 'controls', had $80 \%$ power to detect a difference in survival between the 'high' and the 'low' risk groups at the level of $\mathrm{HR}=1.81$ with 0.05 alpha value.

The gene expression omnibus (GEO) data repository contains the biological experiment (GSE63119) that could be used to confirm the expression of miRNAs constituting the prognostic signature. In GSE63119 series it was possible to examine miRNA expression values (obtained by Illumina sequencing) of $50 \mathrm{CC}$ patients with and without metachronous metastases [30]. In this paper, reads for all isoforms of a given miRNA were counted and $\log 2$ transformed as a basis for miRNA expression estimation. Indirect validation of prognostic value of the expression of miRNAs from the signature was carried out through hierarchical clustering of original and external dataset, and by building logistic regression model on external data set.

To find miRNAs that were expressed only in CC or NCM samples, we discretised the raw $\mathrm{Ct}$ values to the following expression categories: (1) no amplification signal: $\mathrm{Ct} \geq 35$, (2) expression: $\mathrm{Ct}<35$. Discretised, nominal scale data derived from paired samples of NCM and CC were analysed by the McNemar test. For the comparison of miRNA expression between $\mathrm{CC}$ and NCM Ct values for target miRNAs were normalised according to the proposed method 
described in the preceding section. The expression of miRNAs obtained with the $\Delta \mathrm{Ct}$ method was analysed using the paired sample $t$ test. Applying a condition on miRNA to be significantly differentiating between CC and NCM samples in both the original and validation experiments is very conservative, especially in case of small sample sizes. As an alternative the selection algorithm substitutes set of $p$ values by one $p$ value, named combined $p$ value. Combined $p$ value was calculated using the weighted $\mathrm{Z}$ test that requires onesided test $p$ values [31]. When finding one-sided $p$ values for independent experiment, we could directly include the information about the required increase/decrease of miRNA expression observed in original dataset.

ArrayExpress repository contains the data from a biological experiment that could be used to validate miRNAs found to have an amplification signal only in NCM or CC. In E-GEOD-46,622 experiment authors examined miRNA expression values by Illumina sequencing of matched benign, primary tumour and metastatic tissues of eight colorectal cancer patients [32]. To obtain a measure of miRNA expression, reads on target regions were counted and $\log 2$ transformed. Due to the high rate of false positive results in the low range of expression for sequencing experiments, we analysed the distribution of $\log 2$ signal using Gaussian mixture model and k-means algorithm and found the threshold value for expression signal [33]. The values of log2 read counts lower than the estimated threshold were considered as having no amplification signal.

\section{Results}

A total of 85 patients were studied, including 40 who did and 45 who did not develop distant metastases for at least 4 years, with a median follow-up of 3.9 and 5.8 years, respectively. The sites of metastases included lungs, liver, bones and skin. Major clinical characteristics in relapsed and non-relapsed groups did not differ significantly, except for stage (more stage I patients in the control group; Table 1). Out of 754 analysed miRNAs, $159(21 \%)$ with $\mathrm{Ct}<40$ of $0-5 \%$ were considered expression negative. A total of 465 miRNAs $(62 \%)$ were expressed in at least $25 \%$ of all samples, 398 miRNAs (53\%) in at least $50 \%$ of samples, 340 miRNAs ( $45 \%$ ) in at least $75 \%$ of samples and 99 miRNAs (13\%) in all samples. Expression of 229 miRNAs $(30 \%)$ was present in more than $95 \%$ of samples. These miRNAs were subjected to the process of imputation and further analysed. The RNA quality was typical of FFPE samples and the Ct values for RNAs chosen for normalisation are shown in Appendix A, Table 1 in Supplementary material.

\section{MiRNA expression and distant metastasis free survival}

In a univariate Cox regression model, there was a statistically significant association between low expression of miR-1300 and miR-939 and a shorter DMFS (after correction for multiple comparisons, $p=0.049$ ). In the multivariate Cox prognostic models including miRNA expression, $\mathrm{pT}$ stage and tumour grade, low expression of miR-939 [p = 0.011, HR 1.53 (95\% CI 1.10-2.12)] and pT stage [p $=0.05$, HR 2.68 (95\% CI 1.00-7.19)] were independently correlated with DMFS. The comparison of miRNAs median expression between the groups of patients with and without disease recurrence did not result in significant differences after correction for multiple testing. In order to identify miRNAs potentially involved in metastases formation, we searched for miRNAs with expression that was simultaneously correlated with DMFS (unadjusted Cox test $p<0.05$ ) and was different between the patient groups with and without disease recurrence (unadjusted $\mathrm{U}$

Table 1 Patients' clinic-pathological characteristics

\begin{tabular}{llllll}
\hline Variable & Category & $\begin{array}{l}\text { All patients } \\
(\mathrm{N}=85)\end{array}$ & $\begin{array}{l}\text { Relapsed group } \\
(\mathrm{N}=40)\end{array}$ & $\begin{array}{l}\text { Non-relapsed group } \\
(\mathrm{N}=45)\end{array}$ \\
\hline Sex & Male & 50 & 26 & 24 & 21 \\
& Female & 35 & 14 & 28 & 0.275 \\
Stage & I & 40 & 12 & 17 & 8 \\
& IIA & 45 & 28 & 36 & 0.003 \\
Grade & G1 & 11 & 3 & 1 & $65(42-87)$ \\
& G2 & 70 & 34 & $4.25(4.0-11.9)$ \\
Mean age (range) & G3 & 2 & $67(32-83)$ & $7 / 45$ \\
Median follow-up (range) & Years & $66(32-87)$ & $1.7(0.5-3.08)$ & 0.92 \\
MMR status & Years & $3.17(0.5-11.9)$ & $6 / 40$ & $8.97 \mathrm{e}-14$ \\
\hline
\end{tabular}

MMR - DNA mismatch repair system 
Table 2 MiRNAs expression differences between the groups with and without colon cancer relapse

\begin{tabular}{|c|c|c|c|c|c|c|c|c|c|c|c|c|c|c|}
\hline \multirow[t]{2}{*}{ miRNA name } & \multicolumn{2}{|c|}{ Non-meta $\Delta \mathrm{Ct}$} & \multicolumn{2}{|c|}{ Meta $\Delta \mathrm{Ct}$} & \multirow{2}{*}{$\begin{array}{l}\text { Fold } \\
\text { Change }\end{array}$} & \multirow{2}{*}{$\begin{array}{l}\mathrm{FC} \\
\text { Low CI }\end{array}$} & \multirow{2}{*}{$\begin{array}{l}\text { FC } \\
\text { High CI }\end{array}$} & \multicolumn{2}{|l|}{$\mathrm{U}$ test } & \multicolumn{5}{|c|}{ Cox regression } \\
\hline & Mean & SD & Mean & SD & & & & $\mathrm{P}$ value & FDR & $P$ value & FDR & HR & HR 95 & $\% \mathrm{CIs}$ \\
\hline hsa-miR-1300 & 7.44 & 1.14 & 8.32 & 2.00 & 0.54 & 0.33 & 0.90 & 0.035 & 0.634 & $<0.001$ & 0.044 & 1.411 & 1.173 & 1.697 \\
\hline hsa-miR-939 & 5.27 & 1.28 & 6.28 & 1.84 & 0.50 & 0.30 & 0.81 & 0.014 & 0.549 & $<0.001$ & 0.044 & 1.394 & 1.158 & 1.678 \\
\hline hsa-miR-596 & 2.57 & 1.34 & 3.55 & 1.84 & 0.51 & 0.31 & 0.83 & 0.010 & 0.549 & 0.001 & 0.062 & 1.341 & 1.122 & 1.602 \\
\hline hsa-miR-572 (syg) & 8.52 & 1.39 & 9.65 & 1.91 & 0.45 & 0.27 & 0.76 & 0.002 & 0.361 & 0.001 & 0.062 & 1.252 & 1.092 & 1.436 \\
\hline hsa-miR-210 & 3.37 & 1.20 & 4.01 & 1.14 & 0.64 & 0.45 & 0.92 & 0.012 & 0.549 & 0.012 & 0.306 & 1.380 & 1.073 & 1.774 \\
\hline hsa-miR-1303 & 7.80 & 1.30 & 8.73 & 1.93 & 0.52 & 0.32 & 0.87 & 0.040 & 0.634 & 0.013 & 0.306 & 1.222 & 1.043 & 1.433 \\
\hline hsa-miR-422a & 5.34 & 1.68 & 6.33 & 2.20 & 0.51 & 0.28 & 0.92 & 0.032 & 0.634 & 0.014 & 0.306 & 1.207 & 1.039 & 1.403 \\
\hline hsa-miR-7\# & 6.37 & 1.55 & 7.11 & 1.58 & 0.60 & 0.37 & 0.97 & 0.037 & 0.634 & 0.021 & 0.306 & 1.219 & 1.030 & 1.442 \\
\hline hsa-miR-1296 (syg) & 9.90 & 1.69 & 9.08 & 1.49 & 1.76 & 1.09 & 2.86 & 0.027 & 0.634 & 0.022 & 0.306 & 0.772 & 0.618 & 0.964 \\
\hline hsa-miR-185 (syg) & 7.20 & 0.93 & 7.70 & 1.24 & 0.70 & 0.50 & 0.99 & 0.082 & 0.634 & 0.024 & 0.306 & 1.343 & 1.040 & 1.735 \\
\hline hsa-miR-650 & 6.76 & 1.33 & 7.39 & 1.44 & 0.64 & 0.42 & 0.98 & 0.049 & 0.634 & 0.029 & 0.306 & 1.273 & 1.024 & 1.582 \\
\hline has-miR-155 & 2.37 & 1.33 & 3.06 & 1.05 & 0.62 & 0.43 & 0.89 & 0.014 & 0.407 & 0.033 & 0.306 & 1.294 & 1.021 & 1.641 \\
\hline hsa-miR-539 (syg) & 7.31 & 1.99 & 6.53 & 1.24 & 1.71 & 1.05 & 2.81 & 0.212 & 0.699 & 0.062 & 0.393 & 0.819 & 0.664 & 1.010 \\
\hline hsa-miR-135b (syg) & 5.61 & 1.86 & 5.10 & 1.53 & 1.42 & 0.85 & 2.37 & 0.202 & 0.699 & 0.228 & 0.670 & 0.888 & 0.731 & 1.078 \\
\hline
\end{tabular}

Non-meta the group that did not develop relapse, Meta the group that developed distant metastases, $\Delta C t \mathrm{Ct}-\mathrm{geometric}$ mean of $12 \mathrm{Ct}$ normalisers, $F C$ fold change, $F D R$ false discovery rate, $H R$ hazard ratio, $C I$ confidence intervals, syg miRNAs constituting the 5-miRNA signature, SD standard deviation

test $\mathrm{p}<0.05$ ). The expression of miR-1300, miR-939, miR-596, miR-572, miR-210, miR-1303, miR-422a, miR1260, miR-7\#, miR-1296, miR-185, miR-26a-1*, miR-639, miR-650 and miR-155 met both criteria (Table 2). The miRNAs with prognostic or potentially prognostic significance in CC were mostly downregulated in the group with disease recurrence, with the exception of miR-1296 that was upregulated. The full list of correlations of miRNA expression with DMFS (Cox test) and comparisons of miRNAs expression between the relapsed and non-relapsed groups ( $U$ test) are shown in Appendix A, Table 2 in Supplementary material.

An algorithm for prognostic expression model generation resulted in an expression signature including five miRNAs (5-miRNA signature) that were either upregulated (miR-1296, miR-135b and miR539) or downregulated (miR-572 and miR-185) in the CC cases with subsequent relapse. The individual miRNA markers contributed to the signature with the following weights: $\operatorname{logit}(\mathrm{RS})=-3.61$ $-0.72 \times$ miR-135b $+1.45 \times$ miR-185 $-0.59 \times$ miR$539-0.61 \times$ miR-1296 $+0.68 \times$ miR- 572 . This signature's risk score was strongly associated with DMFS [HR 8.4 (95\% CI: 3.81-18.52); $\mathrm{p}<0.004$ ], with sensitivity and specificity of 76 and $87 \%$, respectively (Fig. 1). The median DMFS for the 'high-risk' group was 21 months, and was not reached for the 'low-risk' prognostic group. The 3-year DMFS for the 'high-risk' and the 'low-risk' prognostic groups was 20 and $81 \%$, respectively. The NPV and PPV were 82 and $81 \%$, respectively, and the measure of the model being better than a chance was $\mathrm{p}=6.28 \mathrm{e}^{-08}$ ( $\chi^{2}$ test). In the multivariate Cox model, including the risk score, grade (combined grade 1 and 2 vs. grade 3) and pathological stage (pT2 vs. pT3), the risk score was the only variable that significantly correlated with DMFS [HR 8.91 (95\% CI 3.69-21.48]. Further, the miRNA expression signature was cross-validated in a leave-one-out analysis, yielding sensitivity and specificity of 74 and $78 \%$, respectively. The risk score was also correlated with the overall survival [HR $4.82(95 \% \mathrm{CI}$ : 2.15-10.77); $\left.\mathrm{p}=1.24 \mathrm{E}^{-04}\right]$.

\section{Indirect independent validation of the 5-miRNA prognostic signature}

All miRNAs constituting the 5-miRNA signature were found to be expressed in the external miRNA expression dataset (GSE63119) [30]. Hierarchical clustering using averaged expression signal for each of the five miRNAs from the signature correctly grouped the samples according to their metastasis status in both original and validation experiments, even though the average expression of miR1296 and miR-572 showed some discordances between both datasets (Fig. 2). Expression value of five miRNAs in GSE63119 experiment was obtained in a different highthroughput platform than in the original set, and thus it was not possible to apply directly the 5-miRNA signature with the estimated model coefficients. However, by performing model building with the same rules that were applied to the original data set, we obtained a model that could distinguish relapsed and non-relapsed patients in the independent 

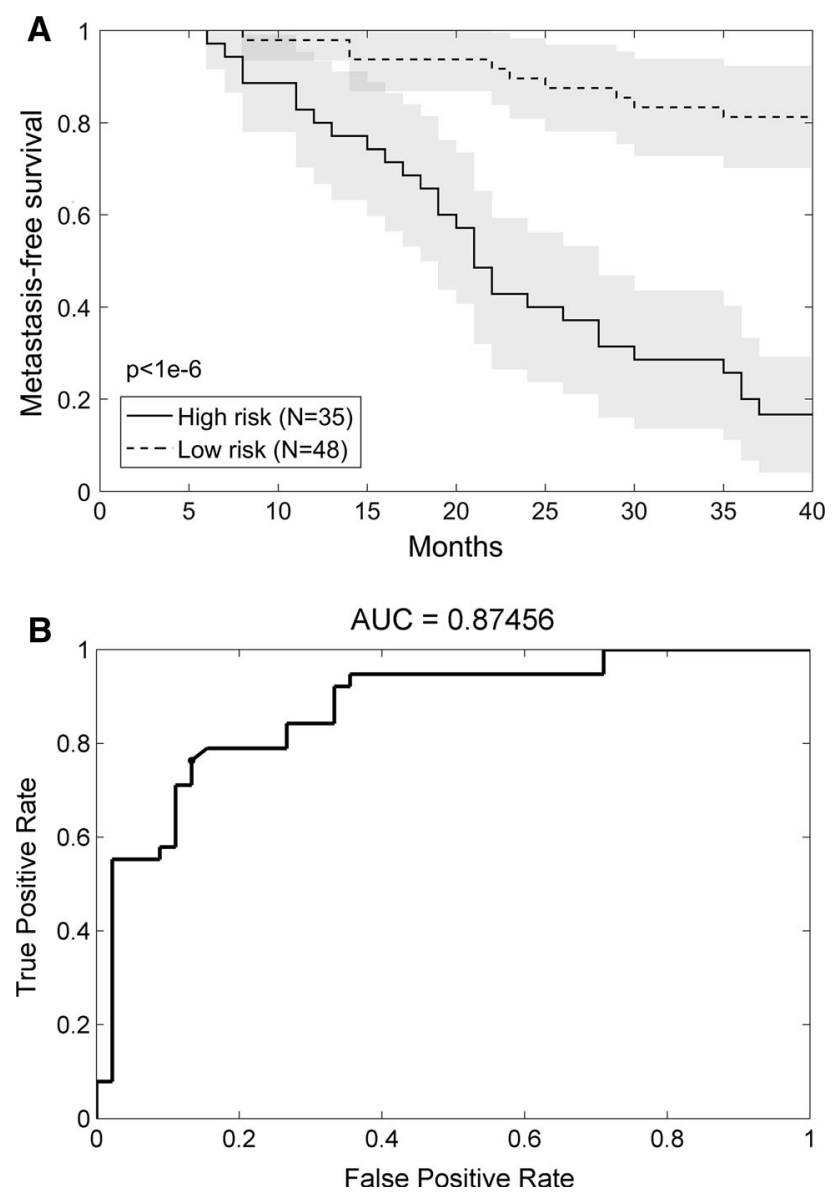

Fig. 1 Metastasis-free survival according to the 5-miRNA expression signature (RS threshold $=0.5$ ) (a) and the area under the curve for estimating 3 year metastasis-free survival using the 5-miRNA expression signature $(\mathbf{b})$

data set. Except for miR-1296, all miRNAs showed the pattern of association of expression signal (upregulation or downregulation) with metastasis status as in the original model. Created model was associated with metastasis status $(\mathrm{AUC}=0.673)$ with sensitivity and specificity of 96 and $27 \%$, respectively. The NPV and PPV were 88 and $55 \%$, respectively.

\section{MiRNA expression according to tumour grade, stage and MIS (microsatellite instability) status}

Out of 14 miRNAs with different expression according to histological tumour grade (p.unadjusted $<0.05$, $\mathrm{U}$ test; Appendix A, Table 3 in Supplementary material), only miR-124 and miR-1243 had higher expression in grade 2 or 3 versus grade 1 tumours. There were 21 miRNAs with expression lower in pT3 versus pT2 tumours (p.unadjusted $<0.05$, U test; Appendix A, Table 3 in Supplementary material). However, none of these differences reached significance after correction for multiple testing.

MMR-deficiency phenotype (dMMR) was present in 13 cases $(15 \%)$, and the remaining 72 cases (85\%) were MMR-competent (cMMR). The frequency of distant metastases in these groups was 53 and $58 \%$, respectively $(\mathrm{p}=0.75)$. Expression of miR-592 was significantly higher in cMMR compared to dMMR CC with the fold change of $11.8 \times(\mathrm{U}$ p.adjusted $=0.0021)$.

\section{MiRNA expression in colon cancer versus normal colon mucosa}

MiRNA expression was compared between the $\mathrm{CC}$ and NCM sample pairs from $14 \mathrm{CC}$ patients. By analysing discretised data we found the expression of 30 miRNAs present specifically in primary tumours, e.g. miR-888, miR-523, miR-18b, miR-302a, miR-339-5p, miR-423-5p, miR-582-3p, miR-1243 ( $\mathrm{p}<0.05)$ and the expression of miR-299-5p and miR-1262 specific to NCM $(\mathrm{p}<0.05)$ (Fig. 3). In the independent dataset (E-GEOD-46622) we found reads for 18 out of 32 miRNAs (56\%) differently expressed between $\mathrm{CC}$ and NCM in the original data set [32]. Eight reads for a given miRNA was a threshold for
Fig. 2 Results of hierarchical clustering of average expression data for miRNAs constituting the 5-miRNA prognostic signature in two independent experiments: the original data set and GSE63119 data set. Expression values measured in two different platforms were integrated using the $\mathrm{z}$-score transformation. The clustering was performed on average signal from 'relapsed' and 'nonrelapsed' samples in both experiments with Euclidean distance metric

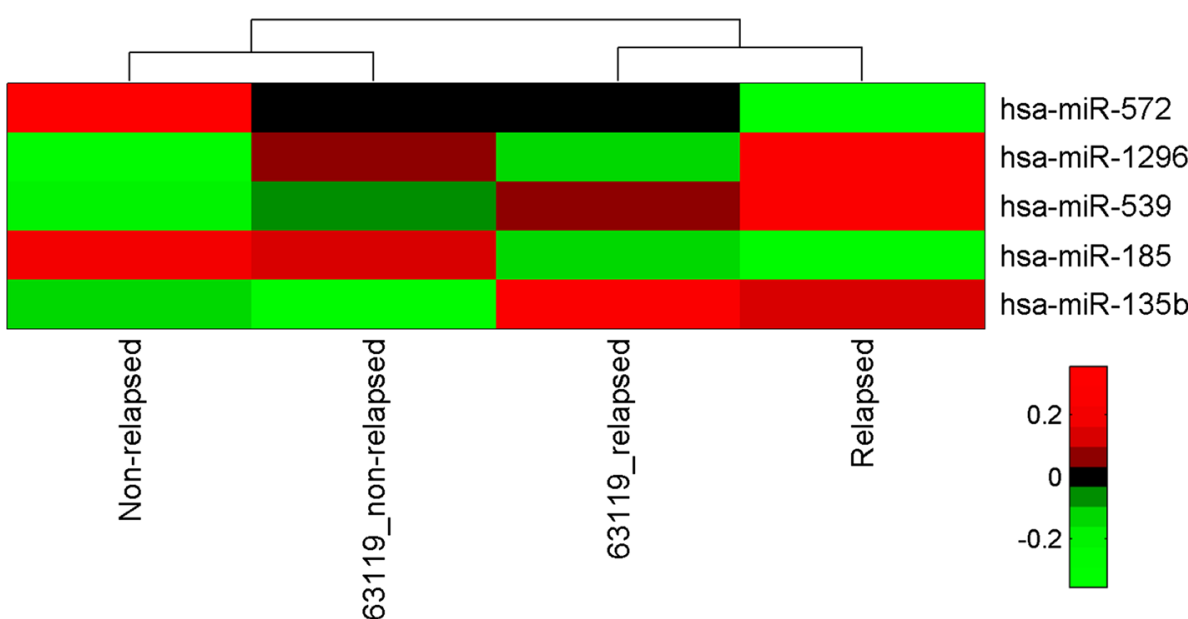



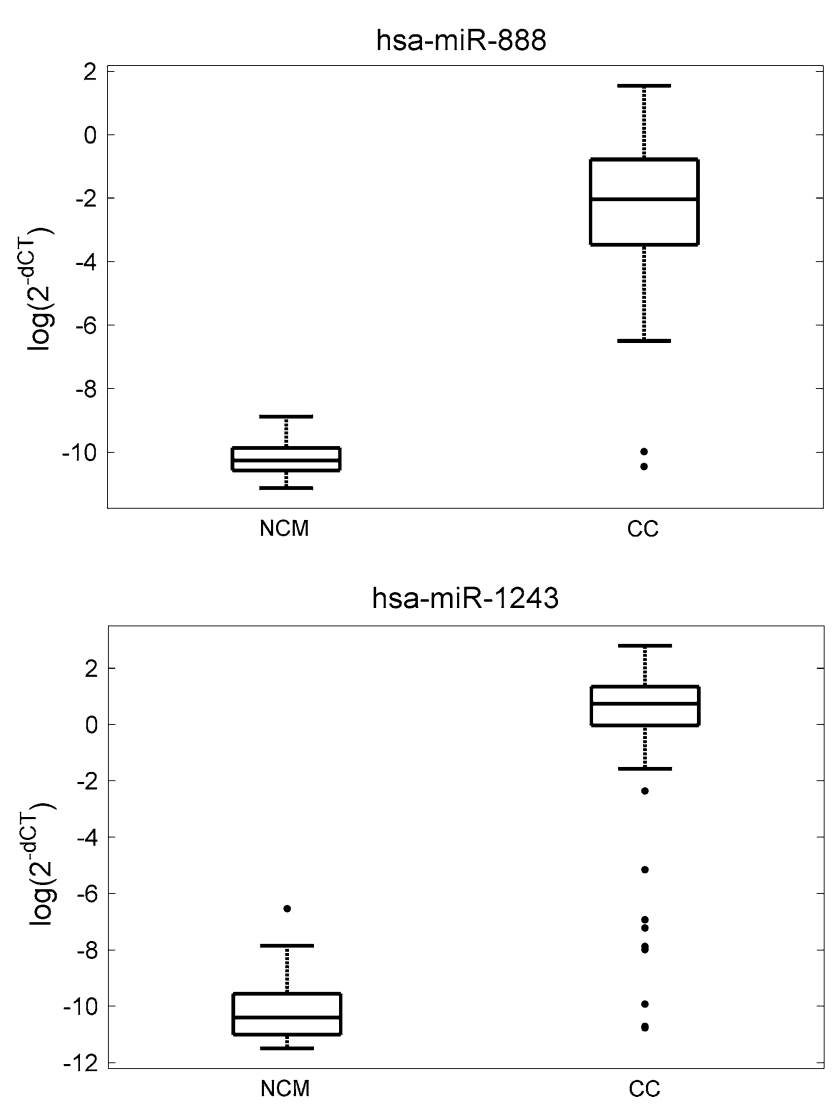

Fig. 3 Expression of miR-888 and miR-1243 in normal colon mucosa (NCM) and in colon cancer (CC)

the expression signal positivity that corresponded to the threshold value of 3.048 in $\log 2$ scale found by using mixture model. By combining $\mathrm{p}$ values we found eight miRNAs significantly differentially expressed between CC and NCM common for both experiments, among which seven were tumour specific, i.e. miR-181c, miR-182, miR32, miR-577, miR-301b, miR-656, miR-372 ( $<<0.05)$ and miR-1262 was expressed specifically in NCM $(\mathrm{p}<0.05)$.

By applying paired $t$ test on $\Delta \mathrm{Ct}$ values we found 282 miRNAs that discriminate NCM and CC samples, among which 112 miRNAs with higher expression in CC samples, e.g. miR-888, miR-21, miR-582-3p, miR-523, miR-520c$3 p(p<0.05)$ and the remaining miRNAs with higher expression in NCM samples, e.g. miR-1262, miR-190b, miR-299-5p, miR-593, miR-633 ( $\mathrm{p}<0.05$ ). In the independent dataset (E-GEOD-46622) we found reads for 77 miRNAs $(27 \%)$ from 282 discriminating CC and NCM samples. Among them there were five miRNAs that discriminated CC and NCM with the same profile of expression (increase/decrease) as in the original data set, i.e. miR31, miR-192, miR-21, miR-145, miR-375 ( $\mathrm{p}<0.05$ ). By combining $\mathrm{p}$ values we found 63 miRNAs significantly discriminating between $\mathrm{CC}$ and NCM common for both experiments, among which 30 were specific for tumours, e.g. miR-21, miR-31, miR-106b, miR-17, miR-135b $(\mathrm{p}<0.05)$ and 33 were specific for NCM, e.g. miR-1262, miR-190b, miR-598, miR-195, miR-145 (p < 0.05).

\section{Discussion}

We report here a 5-miRNA expression signature with potentially strong prognostic impact in early stage CC. To increase the robustness of our data, the analyses were limited to homogeneous groups of patients (pT2N0 and pT3N0 primaries) who underwent adequate radical surgery and had full follow-up data for at least 4 years. The study findings were corroborated in silico in an independent set of CC samples with annotated clinical follow-up information [30]. The signature is based on solid normalisation strategy that can be a reference for future prognostic studies.

The function of miRNAs constituting the signature is being increasingly recognised. Valeri et al. [34] previously reported negative prognostic significance of miR-135b overexpression in CC. Several oncogenic pathways (e.g. $A P C / W n t / \beta$-catenin and PI3KCA) converge on miR-135b, causing its progressive upregulation [34]. MiR-1296 was found to downregulate tumour suppressor $X A F 1$ in immortalised lymphoblastoid cells [35]. Interestingly, miR539, upregulated in the 'high-risk' early stage CC, targets O-GlcNAcase (OGA), an enzyme that posttranslationally removes O-linked $\beta$ - $\mathrm{N}$-acetylglucosamines residues [36]. In vitro, O-GlcNAcylation enhanced the anchorage-independent growth and invasion of lung and CC cells [37], which is line with our findings. MiR-185 has been associated with tumour suppression function, whereby its low expression confers higher risk of dissemination [38, 39]. We have demonstrated for the first time in $\mathrm{CC}$ an inverse correlation between miR-939 expression and DMFS. The prognostic value of this miRNA was previously reported in stage I squamous cell lung cancer patients [40]. In hepatocytes miR-939 was shown to downregulate human inducible nitric oxide synthase (hiNOS), in a check-andbalance system that protects against undue consequences of excessive hiNOS activity [41]. On the other hand, in CC hiNOS activates $\mathrm{Wnt} / \beta$-catenin pathway by negative regulation of $D K K 1$ [42]. Adverse prognostic impact of miR939 downregulation may be therefore linked to increased nitric oxide synthesis that promotes metastasis formation by boosting $\mathrm{Wnt} / \beta$-catenin signalling.

Our study confirms previously reported association between CC MMR status and miR-592 expression [43]. This concordance between two independent studies indicates their methodological accuracy and underscores important differences in epigenetics of cMMR and dMMR CC. Several novel miRNAs with expression differentiating 
CC from NCM were identified, including miR-888, miR523, miR-18b, miR-302a, miR-423-5p, miR-582-3p and miR-299-5p, and the previously reported tumour specific expression of miR-181c, miR-182, miR-301b, miR-92a-1\# confirmed [19, 44, 45].

The relevance of the above findings is further supported by the use of highly sensitive RT-PCR technique, suitable for capturing subtle differences in miRNA expression that are often undetectable with other platforms, e.g. the next generation sequencing (NGS) [46]. MiRNA-specific reverse transcription and target specific fluorescent probes allowed a high level of specificity of the expression signal. Among limitations of this study is the fact that RT-PCR requires a prior knowledge of sequence to be amplified and therefore does not allow for the discovery of new miRNAs or new variants of known miRNAs [47]. On the other hand, a detection bias of NGS towards miRNAs with uracil-rich sequences and a detection bias of oligonucleotide microarrays towards miRNAs with guanine-rich sequences was recently reported [48]. This study does not address the question of tumour microenvironment or cellular compartment localization of miRNA expression, which can be addressed by the use of in situ hybridization with locked nucleic acid (LNA) probes [49]. Still, in reference to RTqPCR, in situ hybridization is more affected by variations in tissue procurement and fixation procedures [50].

Although this study includes homogeneous and carefully selected group of patients, its limitation is a relatively small sample size and an imbalance between 'relapsed' and 'non-relapsed' groups regarding pT stage. Hence, multiinstitutional independent cohort validation of these results, preferably in the context of prospective study, is warranted to verify clinical utility of our findings.

\begin{abstract}
Acknowledgments We would like to acknowledge all participating surgeons and oncologists from the Medical University of Gdansk and from the Centre of Oncology, Bydgoszcz, Poland, in particular: Maciej Świerblewski, MD, PhD; Jarosław Skokowski, MD, PhD; Jacek Zieliński, MD, PhD; Tomasz Jastrzębski, MD, PhD; Kamil Drucis, MD, PhD; Tomasz Polec, MD; Dariusz Bała MD, PhD; Wojciech Grabczewski MD; Jarosław Jaroszek, MD; Mieczysław Kaźmierczak, MD; Michał Klag, MD; Manuela Las - Jankowska, MD; Janusz Piech, MD; Marek Przybył, MD; Tomasz Staniuk, MD, $\mathrm{PhD}$; Ewa Śrutek, MD, PhD; Wiesław Zmysłowski MD, for provision of pathological specimens and clinical data.
\end{abstract}

Financial Support The study was supported by the Polish Ministry of Science, National Center of Science grant no. 2011/03/b/nz5/00519 OPUS II and partially supported by internal grant of Silesian University of Technology BK/RAu1/2016/10 (JP). Calculations were carried out using GeCONiI computational infrastructure funded by a project no. POIG.02.03.01-24-099/13.

\section{Compliance with ethical standards}

Conflict of interest None declared.
Open Access This article is distributed under the terms of the Creative Commons Attribution 4.0 International License (http://crea tivecommons.org/licenses/by/4.0/), which permits unrestricted use, distribution, and reproduction in any medium, provided you give appropriate credit to the original author(s) and the source, provide a link to the Creative Commons license, and indicate if changes were made.

\section{References}

1. Torre LA et al (2015) Global cancer statistics, 2012. CA Cancer J Clin 65(2):87-108

2. Benson AB et al (2004) American Society of Clinical Oncology recommendations on adjuvant chemotherapy for stage II colon cancer. J Clin Oncol 22(16):3408-3419

3. Gray R et al (2007) Adjuvant chemotherapy versus observation in patients with colorectal cancer: a randomised study. Lancet 370(9604):2020-2029

4. Marsoni, S. and I.M.P.A.o.C.C.T. Investigators, Efficacy of adjuvant fluorouracil and leucovorin in stage B2 and C colon cancer. International Multicenter Pooled Analysis of Colon Cancer Trials Investigators. Semin Oncol, 2001. 28(1 Suppl 1): p. 14-9

5. Bleeker WA et al (2001) Prognostic significance of K-ras and TP53 mutations in the role of adjuvant chemotherapy on survival in patients with Dukes C colon cancer. Dis Colon Rectum 44(3):358-363

6. Chang MH et al (2011) Clinical impact of K-ras mutation in colorectal cancer patients treated with adjuvant FOLFOX. Cancer Chemother Pharmacol 68(2):317-323

7. Chen D et al (2014) BRAFV600E mutation and its association with clinicopathological features of colorectal cancer: a systematic review and meta-analysis. PLoS One 9(3):e90607

8. Derbel $\mathrm{O}$ et al (2013) Impact of KRAS, BRAF and PI3KCA mutations in rectal carcinomas treated with neoadjuvant radiochemotherapy and surgery. BMC Cancer 13:200

9. Donada $\mathrm{M}$ et al (2013) Management of stage II colon cancer-the use of molecular biomarkers for adjuvant therapy decision. BMC Gastroenterol 13:36

10. Thibodeau SN, Bren G, Schaid D (1993) Microsatellite instability in cancer of the proximal colon. Science 260(5109):816-819

11. Merok MA et al (2013) Microsatellite instability has a positive prognostic impact on stage II colorectal cancer after complete resection: results from a large, consecutive Norwegian series. Ann Oncol 24(5):1274-1282

12. Network CGA (2012) Comprehensive molecular characterization of human colon and rectal cancer. Nature 487(7407):330-337

13. Venook AP et al (2013) Biologic determinants of tumor recurrence in stage II colon cancer: validation study of the 12-gene recurrence score in cancer and leukemia group B (CALGB) 9581. J Clin Oncol 31(14):1775-1781

14. Salazar R et al (2011) Gene expression signature to improve prognosis prediction of stage II and III colorectal cancer. J Clin Oncol 29(1):17-24

15. Esquela-Kerscher A, Slack FJ (2006) Oncomirs-microRNAs with a role in cancer. Nat Rev Cancer 6(4):259-269

16. Fabian M, Sonenberg N, Filipowicz W (2010) Regulation of mRNA translation and stability by microRNAs. Annu Rev Biochem 79:351-379

17. Lewis B, Burge C, Bartel D (2005) Conserved seed pairing, often flanked by adenosines, indicates that thousands of human genes are microRNA targets. Cell 120(1):15-20 
18. Szafranska AE et al (2008) Accurate molecular characterization of formalin-fixed, paraffin-embedded tissues by microRNA expression profiling. J Mol Diagn 10(5):415-423

19. Zhang JX et al (2013) Prognostic and predictive value of a microRNA signature in stage II colon cancer: a microRNA expression analysis. Lancet Oncol 14(13):1295-1306

20. Slattery ML et al (2014) An evaluation and replication of miRNAs with disease stage and colorectal cancer-specific mortality. Int J Cancer 137(2):428-438

21. Christensen LL et al (2013) MiRNA-362-3p induces cell cycle arrest through targeting of E2F1, USF2 and PTPN1 and is associated with recurrence of colorectal cancer. Int $\mathrm{J}$ Cancer 133(1):67-78

22. Schee K et al (2013) Deep sequencing the MicroRNA transcriptome in colorectal cancer. PLoS One 8(6):e66165

23. Schepeler T et al (2008) Diagnostic and prognostic microRNAs in stage II colon cancer. Cancer Res 68(15):6416-6424

24. McCall MN et al (2014) On non-detects in qPCR data. Bioinformatics 30(16):2310-2316

25. Andersen CL, Jensen JL, Ørntoft TF (2004) Normalization of real-time quantitative reverse transcription-PCR data: a modelbased variance estimation approach to identify genes suited for normalization, applied to bladder and colon cancer data sets. Cancer Res 64(15):5245-5250

26. Livak KJ, Schmittgen TD (2001) Analysis of relative gene expression data using real-time quantitative PCR and the 2(-Delta Delta C(T)) Method. Methods 25(4):402-408

27. Schmittgen TD, Livak KJ (2008) Analyzing real-time PCR data by the comparative C(T) method. Nat Protoc 3(6):1101-1108

28. May S, Hosmer DW (2004) A cautionary note on the use of the Grønnesby and Borgan goodness-of-fit test for the Cox proportional hazards model. Lifetime Data Anal 10(3):283-291

29. Tibshirani $R$ (1997) The lasso method for variable selection in the Cox model. Stat Med 16(4):385-395

30. Goossens-Beumer IJ et al (2015) MicroRNA Classifier and Nomogram for Metastasis Prediction in Colon Cancer. Cancer Epidemiol Biomarkers Prev 24(1):187-197

31. Zaykin DV (2011) Optimally weighted Z-test is a powerful method for combining probabilities in meta-analysis. J Evol Biol 24(8):1836-1841

32. Röhr C et al (2013) High-throughput miRNA and mRNA sequencing of paired colorectal normal, tumor and metastasis tissues and bioinformatic modeling of miRNA-1 therapeutic applications. PLoS One 8(7):e67461

33. Marczyk M et al (2013) Adaptive filtering of microarray gene expression data based on Gaussian mixture decomposition. BMC Bioinform 14:101

34. Valeri N et al (2014) MicroRNA-135b promotes cancer progression by acting as a downstream effector of oncogenic pathways in colon cancer. Cancer Cell 25(4):469-483
35. Lee JE et al (2011) MicroRNA signatures associated with immortalization of EBV-transformed lymphoblastoid cell lines and their clinical traits. Cell Prolif 44(1):59-66

36. Muthusamy S et al (2014) MicroRNA-539 is up-regulated in failing heart, and suppresses O-GlcNAcase expression. J Biol Chem 289(43):29665-29676

37. Mi W et al (2011) O-GlcNAcylation is a novel regulator of lung and colon cancer malignancy. Biochim Biophys Acta 1812(4):514-519

38. Qadir XV et al (2014) miR-185 inhibits hepatocellular carcinoma growth by targeting the DNMT1/PTEN/Akt pathway. Am J Pathol 184(8):2355-2364

39. Liu $\mathrm{M}$ et al (2011) miR-185 targets RhoA and Cdc42 expression and inhibits the proliferation potential of human colorectal cells. Cancer Lett 301(2):151-160

40. Lu Y et al (2012) MicroRNA profiling and prediction of recurrence/relapse-free survival in stage I lung cancer. Carcinogenesis 33(5):1046-1054

41. Guo $\mathrm{Z}$ et al (2012) miRNA-939 regulates human inducible nitric oxide synthase posttranscriptional gene expression in human hepatocytes. Proc Natl Acad Sci USA 109(15):5826-5831

42. Du Q et al (2013) Nitric oxide production upregulates $W n t / \beta$ catenin signaling by inhibiting Dickkopf-1. Cancer Res 73(21):6526-6537

43. Sarver AL et al (2009) Human colon cancer profiles show differential microRNA expression depending on mismatch repair status and are characteristic of undifferentiated proliferative states. BMC Cancer 9:401

44. Tsuchida A et al (2011) miR-92 is a key oncogenic component of the miR-17-92 cluster in colon cancer. Cancer Sci 102(12):2264-2271

45. Neerincx M et al (2015) MiR expression profiles of paired primary colorectal cancer and metastases by next-generation sequencing. Oncogenesis 4:e170

46. Pritchard CC, Cheng HH, Tewari M (2012) MicroRNA profiling: approaches and considerations. Nat Rev Genet 13(5):358-369

47. Metzker ML (2010) Sequencing technologies-the next generation. Nat Rev Genet 11(1):31-46

48. Backes $\mathrm{C}$ et al (2016) Bias in high-throughput analysis of miRNAs and implications for biomarker studies. Anal Chem 88(4):2088-2095

49. Jørgensen S et al (2010) Robust one-day in situ hybridization protocol for detection of microRNAs in paraffin samples using LNA probes. Methods 52(4):375-381

50. Nuovo GJ (2008) In situ detection of precursor and mature microRNAs in paraffin embedded, formalin fixed tissues and cell preparations. Methods 44(1):39-46 